\title{
Observation of Attraction between Dark Solitons
}

\author{
Alexander Dreischuh, ${ }^{1,2,3}$ Dragomir N. Neshev, ${ }^{1}$ Dan E. Petersen, ${ }^{2}$ Ole Bang, ${ }^{4}$ and Wieslaw Krolikowski ${ }^{2}$ \\ ${ }^{1}$ Nonlinear Physics Centre, Research School of Physical Sciences and Engineering, Canberra ACT 0200, Australia \\ ${ }^{2}$ Laser Physics Centre, The Australian National University, Canberra ACT 0200, Australia \\ ${ }^{3}$ Department of Quantum Electronics, Faculty of Physics, Sofia University, Sofia, Bulgaria \\ ${ }^{4}$ COM DTU Department of Communications, Optics \& Materials, Technical University of Denmark, \\ DK-2800 Kongens Lyngby, Denmark
}

(Received 21 March 2005; published 1 February 2006)

\begin{abstract}
We demonstrate a dramatic change in the interaction forces between dark solitons in nonlocal nonlinear media. We present what we believe is the first experimental evidence of attraction of dark solitons. Our results indicate that attraction should be observable in other nonlocal systems, such as Bose-Einstein condensates with repulsive long-range interparticle interaction.
\end{abstract}

DOI: 10.1103/PhysRevLett.96.043901

PACS numbers: 42.65.Sf, 42.65.Jx, 42.65.Tg

It is commonly accepted that solitons, i.e., localized waves propagating without changing their shape, are ubiquitous in nature [1] and are native to many diverse systems like plasmas, molecular chains, spin waves, atmospheric physics, superfluidity, nonlinear optics, and Bose-Einstein condensates (BECs). A soliton can form when the dispersive and/or diffraction processes associated with the finite size of the wave are counterbalanced by the wave selfinduced change of the properties of the medium. In the context of BECs, for example, the soliton represents a coherent excitation of a matter wave [2], whereas in optics it is a localized light beam or pulse [3]. The continuous interest in solitons is stimulated by their unique collisional properties; i.e., they behave like particles displaying forces in their mutual interaction. Furthermore, the fundamental features of their interaction are of rather universal character. Thus, for example, matter wave solitons interact basically in the same way as optical or plasma solitons.

There are two fundamental types of solitons: bright, in the form of a localized structure [4] and dark, in the form of a localized dip on a plane-wave background [5-10]. Already early studies pointed out a distinctive difference between the interaction of bright and dark solitons. Bright solitons can either attract or repel depending on their relative phase. The phase between dark solitons is fixed as they are formed on a common background wave and they are believed to always repel [5,11-13]. This fact imposes a fundamental limit on the applicability of dark solitons and all existing soliton applications are currently based only on bright solitons.

Recently we predicted theoretically [14] that the nature of dark soliton interaction may change drastically in the presence of a nonlocal response of the material. In this Letter we report the first experimental observation of attraction between spatial dark solitons in any physical system. Our findings open new possibilities for control of the interaction between dark solitons, which we believe will revive the interest towards them and will allow for their broader applicability.
In nonlocal nonlinear media the nonlinear response induced at a certain point is carried away to the surrounding regions. In this way a narrow localized wave can induce a spatially broad response of the medium [15]. Spatial nonlocality is an inherent property of many physical systems. It often results from transport processes, such as atomic diffusion [16] or heat conduction [17]. Spatial nonlocality is also natural for media with a long-range interparticle interaction including, for instance, dipolar BECs [18], or nematic liquid crystals with long-range molecular reorientational interactions [19]. It appears that nonlocality leads to novel phenomena of generic nature. For instance, it may promote modulational instability in self-defocusing media $[20,21]$, as well as suppress wave collapse and stabilize multidimensional solitons in self-focusing media [22-25]. Nonlocal nonlinearity may even describe parametric wave mixing [26]. Furthermore, nonlocality significantly changes bright soliton interaction [27].

To test experimentally how the nonlocality affects the forces acting between dark solitons, we considered the propagation of an optical beam in a weakly absorbing liquid. Light absorption increases the temperature of the liquid and subsequently decreases its density and refractive index, resulting in a defocusing nonlinearity. In addition, heat conduction leads to a temperature and consequently refractive index profile much wider than the light beam itself, indicating the inherently nonlocal character of the thermal nonlinearity. The choice of the liquid medium provides additionally the advantage to monitor the beam profile along the entire propagation without destructive intervention to the material.

The propagation of an optical beam along the $z$ axis of a weakly absorbing liquid is described by the system of normalized equations [17] for the slowly varying field amplitude $\psi(x, z, t)$

$$
\frac{\partial \Delta n}{\partial t}-\frac{\partial^{2} \Delta n}{\partial x^{2}}=-\alpha|\psi|^{2}
$$




$$
i \frac{\partial \psi}{\partial z}+\frac{\partial^{2} \psi}{\partial x^{2}}+\Delta n(I) \psi+i \alpha \psi=0
$$

Here the longitudinal $z$ and transverse $x$ coordinates are scaled by the wave vector $k=2 \pi n_{0} / \lambda(z \rightarrow k z / 2, x \rightarrow$ $k x$ ). The parameter $\alpha$ (scaled as $\alpha \rightarrow \alpha / k$ ) represents the linear absorption. Time in Eq. (1) is normalized to $k^{2} D$, where $D$ is the thermal diffusivity of the medium, and $\lambda$ is the laser wavelength. Equation (1) is a heat equation describing the temporal and spatial dynamics of the refractive index change of the medium $\Delta n(I)$ induced by a heat source in the form of a beam with intensity $I(x, z, t)=$ $|\psi(x, z, t)|^{2}$. Equation (2) is a nonlinear Schrödinger equation governing the evolution of the beam amplitude in the presence of nonlinear refractive index change. We solved these equations numerically using a split-step Fourier method for the propagation equation and a finite difference method for the heat equation. For simplicity we assumed unrestricted heat flow in the transverse direction. Using typical thermal parameters of mineral oils yields the diffusion coefficient $D=10^{-7} \mathrm{~m}^{2} / \mathrm{s}$ and linear index $n_{0}=$ 1.5 for $\lambda=0.532 \mu \mathrm{m}$. The absorption coefficient is set to $\alpha=0.01 \mathrm{~cm}^{-1}$ which results in $10 \%$ power loss over $50 \mathrm{~mm}$ of propagation. As an initial condition we used a broad Gaussian beam of peak intensity $I_{0}$ and full width at half maximum (FWHM) $2.8 \mathrm{~mm}$, with two closely placed $\pi$ phase jumps. Such initial conditions result in the formation of two "black" solitons with a zero individual transverse velocities [28].

An example of steady-state trajectories of two adjacent solitons separated by a distance of $60 \mu \mathrm{m}$ is shown in Fig. 1(a). The inward bending of the trajectories clearly indicates the presence of attractive forces. For longer propagation the separation between the two solitons oscillates, leading to formation of an oscillatory-type bound state. This behavior strongly differs from the interaction of dark solitons in local nonlinear media, where the soliton trajectories diverge due to repulsion.
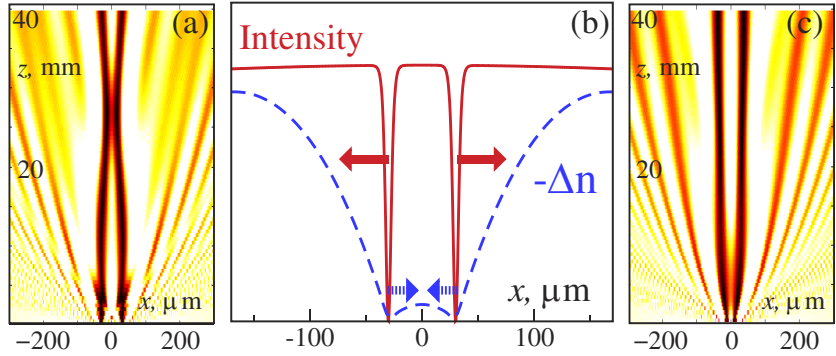

FIG. 1 (color online). (a) Trajectories of two attracting dark solitons separated at $60 \mu \mathrm{m}$. (b) Initial intensity (solid line) and corresponding index structure (dashed line) generated by the nonlocal nonlinear response, giving rise to attractive (dashed arrows) and repulsive forces (solid arrows) on the solitons. (c) Trajectories of two closely spaced dark solitons $(20 \mu \mathrm{m})$ with dominating repulsive interactions.
The physics of this interaction can be intuitively explained as follows. The two close dark solitons with an intensity profile depicted in Fig. 1(b) always try to repel because of local refractive index drop in the overlapping region (repulsive forces are indicated as solid arrows). However, in a nonlocal medium these solitons induce also a large scale change in the refractive index in the form of a broad trapping potential [Fig. 1(b) (dashed curve)]. This potential provides an attractive force (indicated by dashed arrows) which counteracts the natural repulsion of the solitons. Ultimately, the interplay between these two forces will determine the outcome of the interaction. While repulsion prevails for close initial separations and the solitons diverge as depicted in Fig. 1(c), its strength decreases with increase of the separation, allowing the nonlocality-mediated attraction to become dominant at larger separations.

The spacing between the solitons obviously determines the shape of the resulting potential, and subsequently the strength of their interaction. In Fig. 2(a) we present the numerically determined separation between the two dark solitons, after propagation of $50 \mathrm{~mm}$, as a function of their input spacing for low (linear regime - squares) and high intensity (nonlinear regime-circles) of the background beam. For initial spacings larger than $180 \mu \mathrm{m}$ the final separation is close to that of the linearly diffracting dark beams as the nonlocality does not contribute appreciably to the already weak interaction. The most interesting regions in Fig. 2(a) correspond to closely spaced solitons $(20-180 \mu \mathrm{m})$. It is evident that in this region the separation between the solitons is a nonmonotonic function of the initial spacing, being smaller than that in the linear regime. This behavior is a direct manifestation of the nonlocality induced attractive forces. For closely spaced initial dark notches $(20-50 \mu \mathrm{m})$, the generated solitons strongly repel and their final separation is larger than the initial one. In

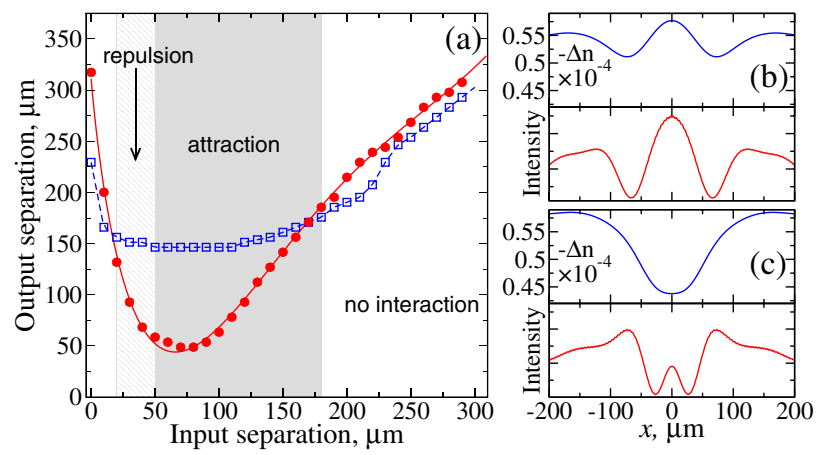

FIG. 2 (color online). (a) Calculated output separation $(z=$ $50 \mathrm{~mm}$ ) vs initial separation between two dark solitons for low $\left(I_{0}=0.01\right.$ - squares $)$ and high $\left(I_{0}=0.5\right.$-circles $)$ intensity of the background beam. The different regimes of interaction are marked. (b), (c) Output intensity (bottom) and index (top) profiles for two different initial separations-(b) repelling solitons $(20 \mu \mathrm{m})$ and (c) attracting solitons $(90 \mu \mathrm{m})$. 
the case of intermediate separations $(50-180 \mu \mathrm{m})$, the attractive forces can balance the natural repulsion and the solitons exhibit oscillating trajectories. Plots in Figs. 2(b) and 2(c) illustrate the output intensity and the refractive index profiles (which play the role of confining potential) in those two cases. For the diverging solitons the potential has a form of two distinct wells separated by a barrier, whereas attraction occurs when the potential represents a single well.

To investigate experimentally the interaction of dark solitons in a nonlocal nonlinear medium we used the experimental setup shown in Fig. 3. A laser beam from a frequency doubled solid-state laser (Verdi-V, $\lambda=532 \mathrm{~nm}$ ) was expanded by a system of lenses and passed through two closely overlapping microscope glass slides. The slides were subsequently imaged by a telescope onto the input face of a $50 \mathrm{~mm}$ long glass cell. The resulting beam diameter at the cell's input was FWHM $=2.8 \mathrm{~mm}$. The cell was filled with paraffin oil dyed with iodine. The iodine served as a weak absorber of the green light and its low concentration of $0.5 \mathrm{mg} / 1$ ensured that the total absorption in the cell was $\approx 10 \%$. The density of paraffin oil decreases with increasing temperature, thus resulting in a self-defocusing nonlinear response. The two glass slides were tilted at a small angle with respect to the beam and modified the phase structure of the beam such that it results in $\pi$ phase jumps in the beam at the position of the glass edge. The phase modulation also gives rise to amplitude modulation at the front face of the cell as seen in the inset of Fig. 4. The input and the output facets of the cell were imaged onto two CCD cameras by large numerical aperture lenses. The beam evolution along the propagation direction was traced by immersing a translatable mirror into the liquid and visualizing the beam profile at different distances [29]. Because of the finite size of this mirror, however, the first $19 \mathrm{~mm}$ of beam propagation could not be directly accessed.

The input beam phase profile was monitored by interference with a reference plane wave [Fig. 4 (top inset)]. Both phase jumps were set to $\pi$ within the accuracy of our

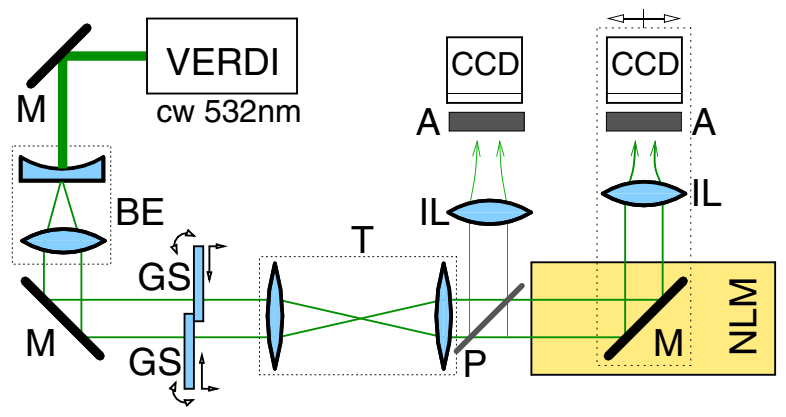

FIG. 3 (color online). Experimental setup: M, mirrors; GS, glass slides; BE, beam expander; $\mathrm{T}$, imaging telescope; $\mathrm{P}$, pellicle; IL, imaging lenses; A, attenuator; NLM, nonlinear medium; CCD, cameras. interferometric measurement $(\sim 10 \%)$. This accuracy was increased by monitoring the beam profile at the far field and setting the intensity in the dark notch to zero. By varying the transverse position of the slides we could change the initial separation between the generated dark notches. A typical intensity profile at a separation of $59 \mu \mathrm{m}$ is shown in [Fig. 4 (bottom inset)]. The width of each notch is $a \sim 18 \mu \mathrm{m}$, which corresponds to $\sim 10$ diffraction lengths of propagation in the nonlinear medium. The individual propagation of each dark notch in the selfdefocusing medium results in formation of a black soliton of zero transverse velocity. The experimental conditions for dark soliton formation were determined by attaining saturation of the so-called soliton constant, $I_{0} a^{2}$, with input beam power [30]. In our experiments this regime was reached at approximately $3 \mathrm{~W}$ of input power. All our measurements were performed at slightly higher power levels $(3.5 \mathrm{~W})$ to assure soliton regime in the presence of interaction and absorption.

When placed sufficiently close, both dark solitons (with parallel initial trajectories) interact during propagation. This influences their output separation, which is compared to the separation at low power. In Fig. 4 we depict the spacing between the dark solitons at the exit of the cell as a function of their initial separation. The dots represent nonlinear regime $(3.5 \mathrm{~W})$, while squares correspond to linear propagation $(10 \mathrm{~mW})$. The measurements quite faithfully reproduce the theoretical predictions. For large initial spacings $(>180 \mu \mathrm{m})$ the solitons are weakly interacting. Their separation in the nonlinear regime, however, is affected by the background beam broadening, resulting in increased separation compared to the linear regime. For initial separations of $20-180 \mu \mathrm{m}$ the final spacing between the

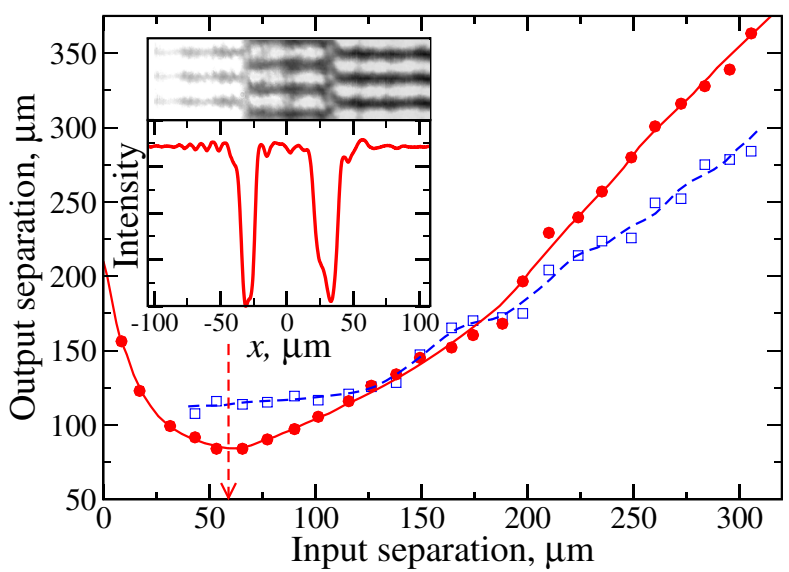

FIG. 4 (color online). Measured output distance between two dark solitons as a function of their initial separation. Dots-nonlinear regime $(3.5 \mathrm{~W})$; squares - linear propagation $(10 \mathrm{~mW})$. For small initial separations the output dark notches are practically not detectable in the linear regime. Inset-interferogram reflecting the initial phase and intensity profile of solitons separated by $59 \mu \mathrm{m}$. 


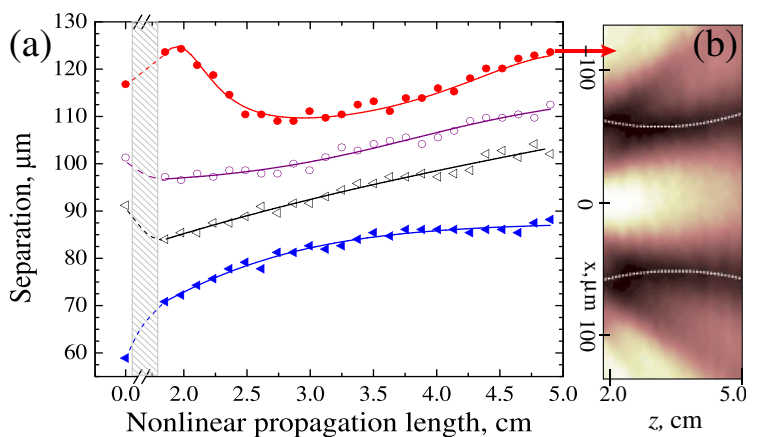

FIG. 5 (color online). (a) Measured output separation of interacting nonlocal dark solitons in thermal medium as a function of the propagation distance for input separation of $117 \mu \mathrm{m}-$ closed circles; $101 \mu \mathrm{m}$-open circles; $91 \mu \mathrm{m}$-open triangles; $59 \mu \mathrm{m}$-closed triangles. (b) Measured trajectories of nonlocal solitons for initial separation of $117 \mu \mathrm{m}$.

solitons is less than in the linear regime. This unambiguously indicates the presence of attractive forces counteracting the natural repulsion of solitons. Moreover, for input spacings of $115-200 \mu \mathrm{m}$ the final separation is actually smaller than the initial one. It should be noted that the separations recorded in the nonlinear regime are detected with high accuracy $(\sim 1 \mu \mathrm{m})$ due to the localization of the dark notches. In the linear regime the determination of the central position of the dark notch is affected by notch broadening and reshaping. The comparison of these two cases, however, gives us the information about the range of initial spacing corresponding to strong attraction.

A clearer picture of soliton interaction can be obtained by following their trajectories inside the medium. This is illustrated in Fig. 5 where we show the measured separation along the cell for initial spacings of 59, 91, 101, and $117 \mu \mathrm{m}$. For the separation of $59 \mu \mathrm{m}$ the repulsive force is strong and cannot be compensated by the nonlocality induced attraction. Therefore, for this separation the two dark solitons repel. The situation changes drastically for larger initial separations. At a certain distance the solitons actually come closer than their initial separation. This behavior cannot be directly observed for the initial spacings of 91 and $101 \mu \mathrm{m}$ since the decrease in soliton separation occurs at the initial nonaccessible part of propagation. However, it is particularly visible for $117 \mu \mathrm{m}$ spacing. The nonmonotonous character of the trajectories is a direct proof of the interplay of repulsive and nonlocality-mediated attractive forces acting between the solitons. When the attraction dominates, solitons decrease their mutual separation until the repulsion prevails forcing them to move apart. The contour plot in Fig. 5(b) shows the experimentally obtained trajectories in this regime (initial spacing of $117 \mu \mathrm{m}$ ). Dashed lines indicate location of the intensity minima. The inward bending of these trajectories is clearly visible.

In conclusion, we have shown what we believe is the first experimental demonstration of the nonlocality-mediated attraction of dark spatial solitons. Our experimental observations are in good agreement with direct numerical simulations. We believe that our results may be applicable to other physical systems exhibiting nonlocal nonlinear response.

The authors thank Yu. S. Kivshar and A. A. Sukhorukov for valuable discussions. This work was supported by the Australian Research Council. A. D. acknowledges support by the NSF-Bulgaria Grant No. F1303/2003.

[1] M. Remoissenet, Waves Called Solitons (Springer-Verlag, Berlin, 1994).

[2] J. Denschlag et al., Science 287, 97 (2000); K. E. Strecker et al., Nature (London) 417, 150 (2002).

[3] Yu.S. Kivshar and G. Agrawal, Optical Solitons: From Fibers to Photonic Crystals (Academic, New York, 2003).

[4] N. Akhmediev and A. Ankiewicz, Solitons, Nonlinear Pulses and Beams (Chapman \& Hall, London, 1997).

[5] G. Swartzlander et al., Phys. Rev. Lett. 66, 1583 (1991).

[6] D. R. Andersen et al., Opt. Lett. 15, 783 (1990); S. R. Skinner et al., IEEE J. Quantum Electron. 27, 2211 (1991).

[7] P. Emplit et al., Opt. Commun. 62, 374 (1987).

[8] Yu. S. Kivshar and B. Luther-Davies, Phys. Rep. 298, 81 (1998), and references therein.

[9] S. Burger et al., Phys. Rev. Lett. 83, 5198 (1999).

[10] N. P. Proukakis et al., J. Opt. B 6, S380 (2004).

[11] K. J. Blow and N. Doran, Phys. Lett. 107A, 55 (1985).

[12] W. Zhao and E. Bourkoff, Opt. Lett. 14, 1371 (1989).

[13] Yu. S. Kivshar and W. Krolikowski, Opt. Commun. 114, 353 (1995).

[14] N. I. Nikolov et al., Opt. Lett. 29, 286 (2004).

[15] A. Snyder and J. Mitchell, Science 276, 1538 (1997).

[16] D. Suter and T. Blasberg, Phys. Rev. A 48, 4583 (1993).

[17] A. G. Litvak et al., Sov. J. Plasma Phys. 1, 31 (1975).

[18] A. Parola, L. Salasnich, and L. Reatto, Phys. Rev. A 57, R3180 (1998); A. Griesmaier et al., Phys. Rev. Lett. 94, 160401 (2005).

[19] D. W. McLaughlin et al., Physica (Amsterdam) 88D, 55 (1995); G. Assanto and M. Peccianti, IEEE J. Quantum Electron. 39, 13 (2003); C. Conti et al., Phys. Rev. Lett. 91, 073901 (2003).

[20] J. Wyller et al., Phys. Rev. E 66, 066615 (2002).

[21] W. Królikowski et al., J. Opt. B 6, S288 (2004).

[22] S. K. Turitsyn, Theor. Math. Phys. (Engl. Transl.) 64, 797 (1985).

[23] O. Bang et al., Phys. Rev. E 66, 046619 (2002).

[24] T. A. Davydova and A. I. Fishchuk, Ukr. J. Phys. 40, 487 (1995).

[25] D. Briedis et al., Opt. Express 13, 435 (2005).

[26] N. I. Nikolov et al., Phys. Rev. E 68, 036614 (2003).

[27] M. Peccianti et al., Opt. Lett. 27, 1460 (2002).

[28] V. E. Zakharov and A. B. Shabat, Sov. Phys. JETP 37, 823 (1973).

[29] D. Neshev et al., Appl. Phys. B 64, 429 (1997).

[30] A. Dreischuh et al., Phys. Rev. E 60, 6111 (1999). 